\title{
gs \\ Gain recovery dynamics of a terahertz quantum cascade laser
}

\author{
Richard P. Green, ${ }^{1,2, *}$ Alessandro Tredicucci, ${ }^{1}$ Ngui Q. Vinh, ${ }^{3}$ Ben Murdin, ${ }^{4}$ Carl Pidgeon, ${ }^{5}$ \\ Harvey E. Beere, ${ }^{6}$ and David A. Ritchie ${ }^{6}$ \\ ${ }^{1}$ CNR NEST-INFM and Scuola Normale Superiore, Piazza dei Cavalieri 7, Pisa I-56126, Italy \\ ${ }^{2}$ Department of Electronics and Electrical Engineering, University of Glasgow, Glasgow G12 8LT, United Kingdom \\ ${ }^{3}$ FELIX-Free Electron Laser Facility, FOM Institute for Plasma Physics "Rijnhuizen," NL-3430 BE Nieuwegein, The Netherlands \\ ${ }^{4}$ Advanced Technology Institute, University of Surrey, Guildford GU2 7XH, United Kingdom \\ ${ }^{5}$ Department of Physics, Heriot-Watt University, Edinburgh EH14 4AS, United Kingdom \\ ${ }^{6}$ Cavendish Laboratory, University of Cambridge, JJ Thomson Avenue, Cambridge CB3 OHE, United Kingdom
}

(Received 13 March 2009; revised manuscript received 12 June 2009; published 7 August 2009)

\begin{abstract}
The gain recovery time of a bound-to-continuum terahertz quantum cascade laser is measured using a stimulated emission photocurrent technique. At low temperatures a value $\sim 50 \mathrm{ps}$ is found. This tends to decrease with rising temperature, and suggests that class B laser dynamics should be expected from these devices. Both the value and the trend in the gain recovery time are found to be consistent with hopping transport through the miniband. Power-dependent measurements of the photocurrent clearly show the effects of inhomogeneous broadening of the gain transition.
\end{abstract}

DOI: 10.1103/PhysRevB.80.075303

PACS number(s): 42.55.Px, 73.63.Hs, 78.47.jc

\section{INTRODUCTION}

Because of the ultrafast lifetimes of the states involved in the lasing transition, quantum cascade lasers (QCLs) ${ }^{1,2}$ have been predicted to show very different dynamical properties from the more familiar semiconductor laser diodes. In particular, it has been predicted that they should be so-called class A lasers, ${ }^{3,4}$ exhibiting much simpler dynamics than class B devices such as the familiar semiconductor diode lasers.

It is well known that the dynamical properties of a laser are described by a system of three coupled rate equations containing the photon density within the laser cavity, the population inversion and the material polarization. There are three lifetimes associated with these dynamical variables: respectively, the photon lifetime, the gain recovery time $\tau_{g}$, and the dephasing time of the laser transition. Often one or more of these relaxation times will be much shorter than the others, so that the corresponding dynamical variable can be adiabatically eliminated, simplifying the rate equations. In class B devices, this is the case for only one of these variables (the polarization), while in class A devices such as the conventional laboratory $\mathrm{He}-\mathrm{Ne}$ laser, the population inversion can also be removed from the appropriate description. As well as the fundamental interest, this matter is of significance for the development of applications involving these devices. It is generally appreciated that the maximum modulation speeds obtainable with class A devices are not limited by relaxation oscillations. Additionally, this also affects the introduction of optical chaos in the devices, since a nonlinear system exhibiting deterministic chaos must be described by at least three dynamical variables; ${ }^{5}$ therefore, in a class B laser, the imposition of only one external variable is required, whereas two are needed to observe the same effect in a class A device. ${ }^{6}$

We present here the results of time-resolved studies of the stimulated emission photocurrent, which were carried out using the Dutch free-electron laser FELIX. These show $\tau_{g} \sim 50 \mathrm{ps}$ and indicate that class B dynamics should be expected from these structures. These data suggest that the electron transport within the miniband does not follow a Bloch model, but is better described by a hopping-based model of sequential scattering events. Power-dependent studies also unambiguously show the inhomogeneous broadening of the gain transition.

\section{EXPERIMENTAL SETUP}

The sample used for these measurements was based on a bound-to-continuum QCL, modified slightly from that reported in Ref. 7 and fabricated into a single metal waveguide structure. Fabry-Perot devices fabricated from the wafershowed good laser performance with threshold current densities $\sim 95 \mathrm{~A} \mathrm{~cm}^{-2}$ and a maximum operating temperature of $70 \mathrm{~K}$. The $250 \mu \mathrm{m}$ by $1.26 \mathrm{~mm}$ sample used for the photocurrent measurements was modified to suppress lasing by polishing the end facets at $45^{\circ}$, preventing optical feedback. This avoided the clamping of population inversion at threshold, allowing measurements over a wider range of inversion levels. During the measurements the device was biased in continuous-wave mode using a constant voltage source, while the photocurrent induced by the FELIX pulses was monitored by measuring the voltage drop across a $1 \Omega$ series resistor.

The FELIX free-electron laser provided a train of $\lambda$ $\sim 94 \mu \mathrm{m}$, transform limited pulses, having a duration of $\sim 10$ ps (spectral width $\sim 1.5 \mu \mathrm{m}$ ). A balanced twobeamsplitter arrangement ${ }^{8}$ was used to split these into two collinearly propagating beams which could be delayed relative to each other using a mechanical delay line, but had the same intensity and polarization. This setup is shown schematically in Fig. 1. The following method was used to measure $\tau_{g}$. By tuning the FELIX wavelength to equal the emission wavelength of the QC structure, the first beam to arrive at the sample depleted the population of the upper laser level 


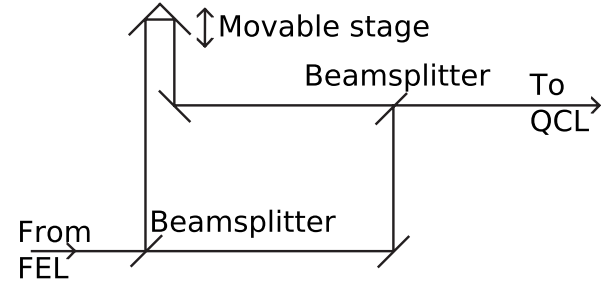

FIG. 1. Schematic showing the two-beamsplitter method used to obtain the variable delay between the two collinear, copolarized pulses used in the experiment. Components solely used to equalize the length of the two paths have been omitted for clarity.

through stimulated emission, causing an instantaneous increase $\delta I$ in the current flowing through the sample. For long time delays between the two optical pulses, the upper laserlevel population had recovered, and so the second pulse to arrive also caused a photocurrent $\delta I$. Obviously the electronics used were too much slow to discriminate between these two pulses, so the measurement indicated a total photocurrent of $2 \delta I$. However, for short time delays, the upper laser-level population was below its steady-state value, resulting in a reduction in the measured photocurrent. By measuring the photocurrent as a function of the optical delay time, it was possible to extract the time needed for the population inversion to recover once it has been depleted. For the non-time-resolved measurements reported here, where the magnitude of the photocurrent was of interest, one of the beams was blocked to avoid interference effects. For all measurements reported here a FELIX wavelength of $\lambda \sim 94 \mu \mathrm{m}$ was used.

\section{PHOTOCURRENT RESPONSE}

\section{A. Bias dependence}

Investigations of the bias dependence of the observed photocurrent were carried out using a single-beam experiment, with a peak FELIX power of $\sim 3 \mathrm{~kW}$. The magnitude of these photocurrents are plotted in Fig. 2(a), together with the $I-V$ characteristic measured from the device [Fig. 2(b)]. The photocurrent data fall into two regimes. At low biases a large photocurrent was observed, which decreased rapidly with bias. The measured photocurrent then reached a minimum, before in the higher current range, within the operating region of the QCL, the signal observed began to increase with applied bias, finally plateauing out, with a possible decrease at the highest current levels. ${ }^{9}$ Comparison with the $I-V$ characteristic of the device, shown in Fig. 2(b) reveals that the changeover between these two regimes occurs at the current where the energy levels of the band structure first become fully aligned in the configuration necessary to support lasing and population inversion is expected to be obtained. The behavior observed here is consistent with a different origin for the photocurrent in the two regions. In the lower current region, before the energy level alignment has taken place, we believe the device to be acting as a QC photodetector, ${ }^{10}$ and the electrons are strongly localized within the ground state of the widest quantum well. The measured signal arises here due to the photoexcitation of an

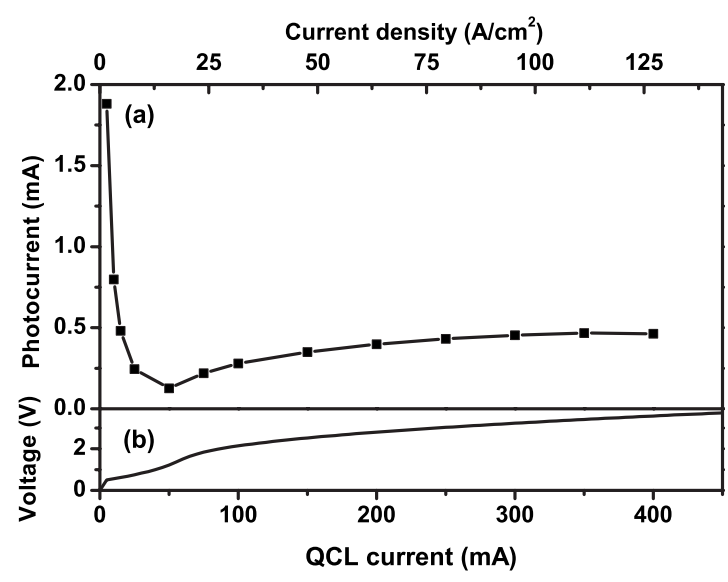

FIG. 2. (a) Photocurrent magnitude measured as a function of the applied bias current, using a FELIX peak power of $\sim 3 \mathrm{~kW}$ at $\lambda \sim 94 \mu \mathrm{m}$. The device temperature was $\sim 20 \mathrm{~K}$. (b) Continuouswave $I-V$ characteristic measured from the device under the same operating conditions.

electron into an excited state, and its subsequent decay into the ground state of the next period of the structure. Schrödinger-Poisson simulations of the structure under zero bias [Fig. 3(a)] show the presence of a large dipole absorptive transition close in energy to the FELIX radiation. However, once the band alignment has taken place, as shown in Fig. 3(b), absorptive transitions close to the laser energy are weak $^{11}$ and the photocurrent is attributed to the stimulated emission process detailed above.

\section{B. Time-resolved measurements}

Two-beam experiments were carried out to measure values of $\tau_{g}$; a typical trace of the measured photocurrent against the time delay between the two pulses is shown in Fig. 4. Because the two beams are of nominally the same power and polarization, the shape of the curve is roughly symmetrical. Nevertheless, data obtained for positive and

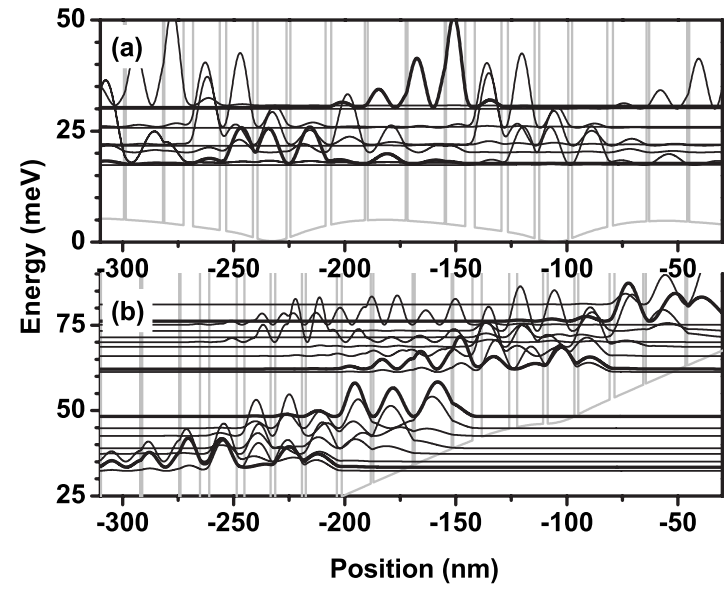

FIG. 3. Calculated band diagrams for the structure under (a) zero bias and (b) expected operating bias, showing $|\Psi|^{2}$ for selected wave functions. Bold lines indicate the energy levels involved in radiative transitions. 


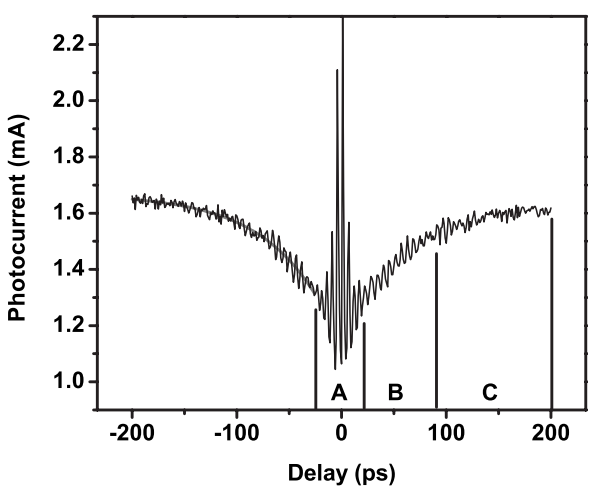

FIG. 4. A typical trace of photocurrent against delay time between the two beams, measured at $300 \mathrm{~mA}$ bias current. The thick gray line shows a single exponential fit to the negative delay data. Letters indicate different regions of the curve as detailed in the text.

negative delay times were fitted separately and the results averaged. The data fall into three different regimes. Close to the zero-delay point, in region A of Fig. 4, high-amplitude oscillations of the photocurrent are observed, as a consequence of interference between the two pulses temporally overlapping on the sample. In region B there are still oscillations in the photocurrent, superimposed on a general trend. Careful study of the data shows that the oscillations in regions $\mathrm{A}$ and $\mathrm{B}$ exhibit slightly different periods, and that the region $\mathrm{B}$ oscillations are not present at the lowest bias currents, when the device is acting as a QC detector. We believe that the origin of these features of the data may lie in the coherent oscillation of electron wave packets across the injection barrier, ${ }^{12}$ induced by the sudden depopulation of the upper laser level. The measured period of these oscillations $(\sim 3 \pm 1.5 \mathrm{ps})$ is in reasonable agreement with the expected $5 \mathrm{ps}$, based on the calculated tunnel coupling of $0.8 \mathrm{meV}$ between the injector and upper laser levels.

Values of $\tau_{g}$ were obtained by fitting the data in regions B and $\mathrm{C}$ to a single exponential curve. The times obtained are plotted as a function of applied bias current in Fig. 5(a). Over the range of currents for which the QCL would be expected to exhibit optical gain, the recovery times obtained remain very similar, $\sim 50 \mathrm{ps}$. In the higher current range this corresponds to the gain recovery time, $\tau_{g}$, whereas at lower currents the structure is effectively acting as a saturable absorber, and the longer times measured relate to the absorption recovery time.

There are two components to $\tau_{g}$ : the time taken for the electron to leave the lower laser level and transit through the miniband, and the tunneling time across the injection barrier. The latter process takes place by resonant tunneling and is expected to occur very rapidly. Although no experimental studies of its value have so far been carried out for $\mathrm{THz}$ QCLs, mid-infrared pump-probe measurements have suggested that transfer across the barrier takes place within 3-4 ps. ${ }^{12}$ The obtained long lifetimes are thus dominated by the transport of the electron within the miniband. Using a $50 \mathrm{ps}$ gain recovery time, and the $123 \mathrm{~nm}$ period of the quantum cascade structure, we estimate the electron drift velocity within the miniband to be $v_{d} \sim 2.5 \times 10^{5} \mathrm{~cm} \mathrm{~s}^{-1}$.

This surprisingly high value for $\tau_{g}$ can be understood in terms of hopping transport through the miniband, where the

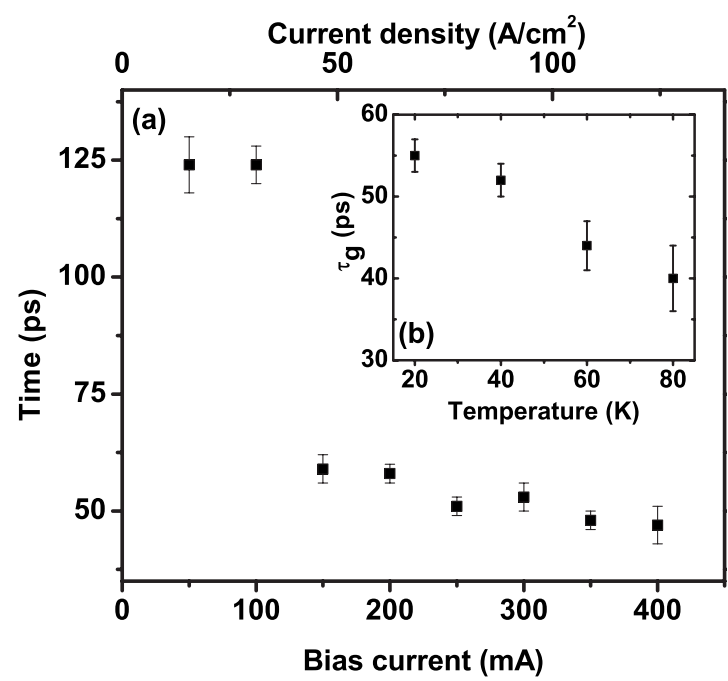

FIG. 5. (a) Gain recovery times measured as a function of the bias current. The measurements were carried out using $94 \mu \mathrm{m}$ laser excitation at a power of $\sim 1 \mathrm{~kW}$. (b) Gain recovery times measured as a function of temperature. A $300 \mathrm{~mA}$ bias current was used, with a FELIX peak power of $\sim 3 \mathrm{~kW}$.

electrons are sequentially scattered between the individual states forming a Wannier-Stark ladder. While there is an extensive literature on this subject, ${ }^{13}$ the majority relates to transport through either very narrow minibands (width $\sim 1 \mathrm{meV}$ ) or to strongly coupled ones with a miniband width greater than the optical phonon energy and so is not directly comparable with our structure. Rott et al. ${ }^{14}$ have calculated the field-dependent drift velocity for a specific structure with a 20-meV-wide miniband. At a comparable electric field of $\sim 2.5 \mathrm{kV} \mathrm{cm}^{-1}$, they predict $v_{d} \sim 10^{5} \mathrm{~cm} \mathrm{~s}^{-1}$, very similar to our measured value. However, there are several differences between these two structures which might modify the mobility. Rather than being uniformly doped, in THz QCLs the dopant atoms are usually concentrated in a single quantum well, set back from the injection barrier to reduce the impurity-induced scattering of electrons out of the upper laser level. In the QCL structure we are considering, the average doping density over the whole period is approximately four times lower than in the structure considered by Rott $e t$ $a l .{ }^{14}$ and so the impurity-induced scattering would be expected to be reduced. In addition, the miniband in our structure is somewhat narrower $(16 \mathrm{meV}$ vs $20 \mathrm{meV})$. Both of these factors would be expected to result in a reduced mobility in the QCL when compared to the superlattice used for their calculations. However, it also has to be remembered that the QCL structure differs from a standard superlattice through not having translational symmetry. In a traditional Wannier-Stark ladder each wave function is mainly localized within a single quantum well, so that the conductance is dominated by $|\Delta \nu|=1$ scattering events, where $\nu$ represents the level index. In contrast, the miniband within a bound-tocontinuum QCL is structured so that at the design field each wave function is delocalized over two or more quantum wells, increasing the probability of $|\Delta \nu|>1$ transitions taking place. This fact would in contrast be expected to increase the drift velocity within the miniband. 
Further evidence for hopping-based transport within the miniband is provided by the temperature dependence of $\tau_{g}$, shown in Fig. 5(b). These were measured at a constant bias current of $300 \mathrm{~mA}$, with a FELIX peak power of $\sim 3 \mathrm{~kW}$, increased above that used for the current-dependent measurements to allow a good signal to noise ratio to be maintained over a wider temperature range. We observe a consistent decrease in $\tau_{g}$ as the temperature is increased, agreeing with the model of transport within the miniband taking place by successive scattering events between states within a WannierStark ladder. In contrast, Bloch transport through the superlattice would be expected to become slower as the temperature is raised, with the mobility being limited by phonon scattering. ${ }^{15}$ We note that recent measurements of $\tau_{g}$ in mid-infrared $(\lambda \sim 4-5.5 \mu \mathrm{m})$ QCLs (Ref. 16) have shown the presence of Bloch transport. These devices were designed with much greater miniband width $(\sim 150 \mathrm{meV})$ than those considered here, which we consider to be the reason for this difference between mid-infrared and THz QCLs.

The measured values of $\tau_{g}$ are about four times longer than the expected $\sim 12$ ps lifetime of a photon within the laser cavity. ${ }^{17}$ We can now compare these values with those relevant for semiconductor laser diodes emitting at telecommunications wavelengths in the near infrared. Since the facet reflectivity, device length, and refractive index are approximately the same, the photon lifetimes for the two types of devices are broadly similar. Recent measurements of the gain recovery time, associated with band refilling in a bulk $\lambda$ $\sim 1.55 \mu \mathrm{m}$ semiconductor optical amplifier, ${ }^{18}$ show a strong bias dependence, with $\tau_{g} \sim 20-160 \mathrm{ps}$, being of a similar order of magnitude to the value reported here for a $\mathrm{THz}$ QCL. This strongly suggests that the dynamic properties to be expected from this QCL design will resemble the class B behavior of semiconductor diode lasers, rather than class A.

The gain recovery time can be expected to vary significantly between different QCL designs. In particular, in QCL designs reliant on optical phonon-assisted depopulation of the lower laser level ${ }^{1,19}$ the energy spacing between the lower laser level and the injector state/upper laser-level doublet in the succeeding period of the structure is greater than an optical phonon energy. In this situation, electrons can rapidly lose energy through LO phonon emission, and the gain recovery time is likely to be correspondingly faster. ${ }^{20}$

\section{Saturation properties}

Measurements were also carried out of the magnitude of the photocurrent response as a function of the FELIX power incident on the sample at a fixed bias current of $300 \mathrm{~mA}$. The results of this are shown in Fig. 6(a). It is well known that the saturation or bleaching of a transition is described by the expression $^{21}$

$$
\Delta I \propto \frac{P}{\left(1+\frac{P}{P_{\text {sat }}}\right)^{m}},
$$

where $\Delta I$ is the induced photocurrent, $P$ is the incident power, and $P_{\text {sat }}$ is the saturation power, at which the saturated absorption coefficient reaches half of its unsaturated

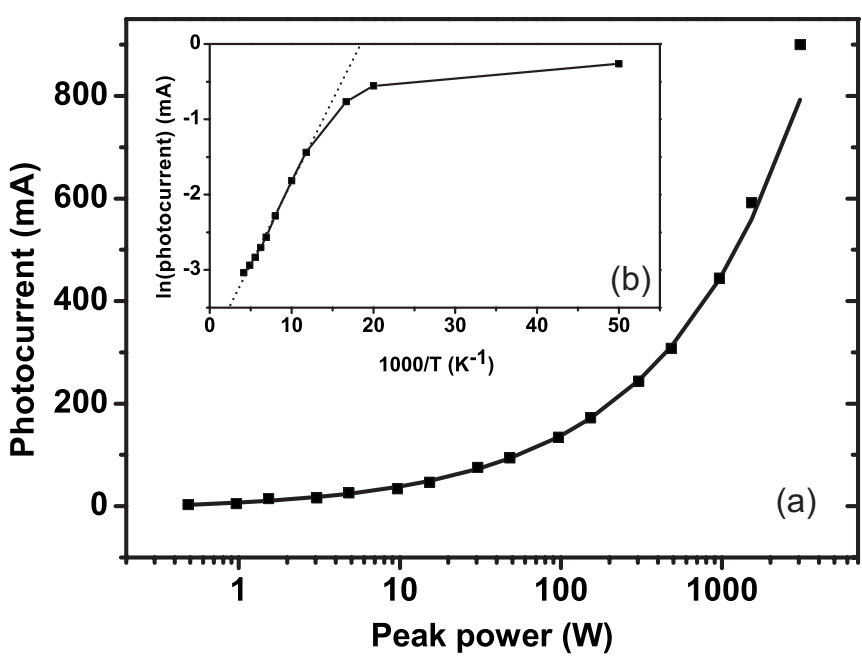

FIG. 6. (a) Symbols represent the measured photocurrent as a function of FELIX peak power, at $\lambda=94 \mu \mathrm{m}, 300 \mathrm{~mA}$ bias current and $20 \mathrm{~K}$ device temperature. The line is a fit to the data using the expression for inhomogeneous broadening and a saturation power of $240 \mathrm{~mW}$. (b) Arrhenius plot of the photocurrent measured as a function of temperature. The same conditions were used, but the FELIX peak power was fixed at $\sim 1 \mathrm{~kW}$. The dashed line represents a fit to the high-temperature data, using an activation energy of $5.5 \mathrm{meV}$.

value. The value of $m$ differs depending on the broadening mechanism applicable for the transition. For a purely homogeneously broadened transition $m=1$, while for inhomogeneous broadening it is equal to 0.5 . The solid line in Fig. 6(a) is the result of a fit to the measured data with the above expression using $m=0.5$, and a saturation peak power of 240 $\mathrm{mW}$. It should be noted that this power represents the FELIX power output rather than the power actually coupled into the device, which will be significantly lower due to the mode mismatch between the large focus provided by the parabolic mirror, and the QCL waveguide mode.

One possible reason for the inhomogeneous broadening observed is due to the diagonal nature in real space of the laser transition, resulting in a strong role for the interface roughness. ${ }^{22}$ However we cannot exclude the possibility of small differences in either the physical structure or electric field experienced by the individual QC active regions, or even variations in the electric field across the laser ridge. The existence of an inhomogeneously broadened gain is potentially highly beneficial for the development of QC amplifiers of terahertz light ${ }^{23}$ since it reduces the adverse effects of gain saturation on the available output power.

\section{Temperature dependence}

Under the same conditions, but with a fixed FELIX power, the temperature dependence of the photocurrent magnitude was investigated. This illuminates a different aspect of QCL operation to the temperature variation in $\tau_{g}$ studied above, since the photocurrent magnitude is sensitive to the population inversion, while the gain recovery time studies give us information about the dynamics of the electrontransport process through the miniband. The magnitude of 
the photocurrent measured at a range of temperatures is shown on an Arrhenius plot in Fig. 6(b). We consider the reduction in the measured photocurrent at high temperatures to be a direct result of the lower population in the upper laser level and consequent reduction in the stimulated emission process responsible for the photocurrent measured in our experiment. An activation energy of $\sim 5.5 \mathrm{meV}$ is obtained from a linear fit to the high-temperature data. Study of the band diagram of the structure under operating bias, as shown in Fig. 3(b) shows that the most likely channel for thermal activation of electrons out of the upper laser level is into the higher lying state localized mainly in the first two wells of the miniband; previous photoluminescence studies of operating QCLs have supported the suggestion of carrier leakage into this state. ${ }^{24}$ The measured activation energy is in reasonable agreement with the calculated position of this level $\sim 7.5 \mathrm{meV}$ above the upper laser level.
In conclusion we have used time-resolved measurements of the stimulated emission photocurrent to measure the gain recovery time in a terahertz quantum cascade laser. These suggest that transport through the miniband takes place by a hopping process, and that these devices should be expected to show class B laser dynamics. The power dependence of the observed photocurrent indicates that the laser transition is likely to be inhomogeneously broadened.

\section{ACKNOWLEDGMENTS}

We gratefully acknowledge the support by the Stichting voor Fundamenteel Onderzoek der Materie (FOM) in providing the required beam time on FELIX and highly appreciate the skilful assistance by the FELIX staff. This work was partially supported by the European Community through the Marie Curie RTN POISE and the IP project TERANOVA. *rgreen@elec.gla.ac.uk

${ }^{1}$ J. Faist, F. Capasso, D. Sivco, C. Sirtori, A. Hutchinson, and A. Cho, Science 264, 553 (1994).

${ }^{2}$ R. Köhler, A. Tredicucci, F. Beltram, H. E. Beere, E. H. Linfield, A. G. Davies, D. A. Ritchie, R. C. Iotti, and F. Rossi, Nature (London) 417, 156 (2002).

${ }^{3}$ R. Paiella, R. Martini, F. Capasso, C. Gmachl, H. Y. Hwang, D. L. Sivco, J. N. Baillargeon, A. Y. Cho, E. A. Whittaker, and H. C. Liu, Appl. Phys. Lett. 79, 2526 (2001).

${ }^{4}$ C. Y. Wang, L. Diehl, A. Gordon, C. Jirauschek, F. X. Kärtner, A. Belyanin, D. Bour, S. Corzine, G. Höfler, M. Troccoli, J. Faist, and F. Capasso, Phys. Rev. A 75, 031802 (2007).

${ }^{5}$ H. Haken, Light: Laser Light Dynamics (North-Holland, Amsterdam, 1985), Vol. 2.

${ }^{6}$ F. Kuwashima, I. Kitazima, and H. Iwasawa, Jpn. J. Appl. Phys., Part 2 37, L325 (1998).

${ }^{7}$ G. Scalari, L. Ajili, J. Faist, H. Beere, E. Linfield, D. Ritchie, and G. Davies, Appl. Phys. Lett. 82, 3165 (2003).

${ }^{8}$ P. Rauter, T. Fromherz, G. Bauer, N. Q. Vinh, B. N. Murdin, J. P. Phillips, C. R. Pidgeon, L. Diehl, G. Dehlinger, and D. Grützmacher, Appl. Phys. Lett. 89, 211111 (2006); H. Schneider, T. Maier, M. Walther, and H. C. Liu, ibid. 91, 191116 (2007).

${ }^{9}$ The photocurrent within these two ranges would be expected to have opposite polarity. However our experimental setup only measured the magnitude of the photocurrent. The saturation of photocurrent observed at high biases is most likely due to the thermal rollover of the device in $\mathrm{cw}$ operation.

${ }^{10}$ D. Hofstetter, M. Beck, and J. Faist, Appl. Phys. Lett. 81, 2683 (2002).

${ }^{11}$ R. P. Green, J.-H. Xu, L. Mahler, A. Tredicucci, F. Beltram, G. Giuliani, H. E. Beere, and D. A. Ritchie, Appl. Phys. Lett. 92, 071106 (2008).

${ }^{12}$ F. Eickemeyer, K. Reimann, M. Woerner, T. Elsaesser, S. Barbieri, C. Sirtori, G. Strasser, T. Müller, R. Bratschitsch, and K. Unterrainer, Phys. Rev. Lett. 89, 047402 (2002).
${ }^{13}$ See, for example, A. Wacker, Phys. Rep. 357, 1 (2002).

${ }^{14}$ S. Rott, N. Linder, and G. H. Döhler, Phys. Rev. B 65, 195301 (2002).

${ }^{15}$ J. F. Palmier, H. Le Person, C. Minot, A. Chomette, A. Regreny, and D. Calecki, Superlattices Microstruct. 1, 67 (1985).

${ }^{16}$ H. Choi, T. B. Norris, T. Gresch, M. Giovannini, J. Faist, L. Diehl, and F. Capasso, Appl. Phys. Lett. 92, 122114 (2008); H. Choi, L. Diehl, Z.-K. Wu, M. Giovannini, J. Faist, F. Capasso, and T. B. Norris, Phys. Rev. Lett. 100, 167401 (2008).

${ }^{17}$ Assuming uncoated facets and mirror losses $\alpha_{w} \sim 10 \mathrm{~cm}^{-1}$ (i.e., a device length just over $2 \mathrm{~mm}$ ).

${ }^{18}$ R. Giller, G. T. Robert, J. Manning, R. P. Webb, and M. J. Adams, Opt. Express 15, 1773 (2007).

${ }^{19}$ B. S. Williams, H. Callebaut, S. Kumar, Q. Hu, and J. L. Reno, Appl. Phys. Lett. 82, 1015 (2003).

${ }^{20}$ Indirectly obtained values for $\tau_{g}$, after the method of G. Scalari et al. [Appl. Phys. Lett. 91, 032103 (2007)], rely on having an accurate value for the sheet doping density $n_{s}$. In order to reproduce the value of $\tau_{g}$ obtained here, we have to use a doping density $\sim 3 \times$ lower than intended; This is consistent with the previous report of $C-V$ measurements of a THz QCL [G. Scalari et al., ibid. 94, 041114 (2009)] which indicate a sheet density $\sim 2.5 \times$ lower than intended. One possible reason for this discrepancy in doping levels could be the compensation of the dopants owing to depletion effects in the thin dopant layers.

${ }^{21}$ A. Siegman, Lasers (Oxford University Press, Oxford, 1986), p. 254.

${ }^{22}$ J. Faist, F. Capasso, C. Sirtori, D. L. Sivco, A. L. Hutchinson, and A. Y. Cho, Nature (London) 387, 777 (1997).

${ }^{23}$ C. Mauro, R. P. Green, A. Tredicucci, F. Beltram, H. E. Beere, and D. A. Ritchie, J. Appl. Phys. 102, 063101 (2007).

${ }^{24}$ M. S. Vitiello, G. Scamarcio, V. Spagnolo, T. Losco, R. P. Green, A. Tredicucci, H. E. Beere, and D. A. Ritchie, Appl. Phys. Lett. 88, 241109 (2006). 